\title{
Pump elements recondition by electrolytic chromium plating
}

\author{
Galia Kokieva $^{1 *}$ and Varvara Dryzianova ${ }^{2}$ \\ ${ }^{1}$ Arctic State Agrotechnological University, 3, Sergelyakhskoe highway $3 \mathrm{~km}, 677007$, Yakutsk, the \\ Republic of Sakha (Yakutia), Russia \\ ${ }^{2}$ North-Eastern Federal University named after M.K. Ammosov, 58, Belinskogo st., 677007, Yakutsk, \\ the Republic of Sakha (Yakutia), Russia
}

\begin{abstract}
Improving the efficiency of maintenance production in agriculture closely connected to the creation and implementation of such methods of repair and recondition of elements that improve the physicomechanical and service properties of wear joint assemblies. The presence of a nitrided case on the surface of elements lays the groundwork for obtaining a complex covering based on chromium nitrides. Electrolytic chromium plating is one of the ways to recondition elements that increase the service life and machines reliability, and reduce maintenance cost. However, the widespread adoption of chromium plating for the recondition of worn elements is hold back by the low productivity of the process. Therefore, the intensification of chromium plating in reconditioning wornout machine elements is an urgent problem. It is advisable to recondition critical parts subjecting to abrasive wear, for example, precision vapors of fuel injection equipment, with chromium plating. However, the low productivity and high-energy consumption of the process require the improvement of the electrolyte in order to increase the current output of chromium and the permissible cathode density. The article describes a method for improving the quality of an electrolyte, a method for increasing its versatility, namely the supplementation of various organic additives and complexing substances into it.
\end{abstract}

\section{Introduction}

To improve the quality of the electrolyte, the increased versatility into it is introduced various organic additives and complexing substances. An increase in the amount of organic additive leads to an increase in the performance of chromium. The manufacturing of replacement parts for agricultural implements requires not only significant labor and energy inputs, but also extra metal consumption (about $50 \%$ of the metal requirement for the making new machines). In melting worn-out parts, non-collectable corrosion loss, burningout, scrap waste are up to $40 \%$. The engineering of renovation of component parts: worn out machine - blast furnace - machine-building plant - consumer - downtime and expensive

*Corresponding author : kokievagalia@mail.ru 
process. Shorter and more progressive manufacturing chain - worn out machine - repair factory - consumer. Component reconditioning requires 20-30 times less metal than manufacturing new duplicate parts. For example, the crankshaft of a Yaroslavl Engine Plant $-24 \mathrm{vol}$. oil engine is made from a blank part weighing $340 \mathrm{~kg}$. About $200 \mathrm{~kg}$ of unavailable alloy steel goes to waste. At the same time, the recondition of the shaft requires max $8 \mathrm{~kg}$ of surfacing wire. The count of making the parts cutting during reconditioning is reduced by 3-5 times compared to the manufacture of new duplicate parts. The effective and paced working of maintenance bases is often hold because of an acute problem of supplying them with duplicate parts and components, deterioration of the train of machines, processing equipment, instrumenting, tools, controlling and verifying instruments. Many maintenance bases have to organize the recondition and making of repair units of a comprehensive range because of the corroded economic ties the cessation of deliveries. In this difficult situation, the problem of assimilation of novel remanufacturing technologies is of particular importance. One of the most important priorities of the repair industry of agricultural implements is to increase the service life of machines and mechanisms $[5,13,14]$.

The service reliability of the elements of the fuel fitment of oil engines largely determines the environmental engine safety, the constancy and operational safety, and its operational and economic parameters. The wear of the pump elements of an oil engine of agricultural implements is the reason for power-supply failure in $30-50 \%$ of all power unit failures. The high pressure in fuel priming, the quality of the materials used in the manufacture of points, the build quality, blocking, etc. cause it.

However, the main reason is in the quality of the diesel oil used, containing solid mechanical particles, which leads to an abrasive wear. A large number of pump elements of oil fuel pumps of trucks' engines are rejected annually in agriculture. These parts reconditioning would significantly reduce the components use and cut repairs costs. There is no rational method of integrated specifications of the technical and economic parameters entirety characterizing agricultural units up to the present. The main operational and economic parameters of the compared units: productivity, labor input, fuel consumption, primary and maintenance and repair costs may vary. In all cases, the machine system that makes the maximum output with the lowest values of the other parameters is acceptable.

It is customary toput value on the economic viability of reconditioning parts by comparing a new part cost and repair maintenance of a worn one. However, the difference of the service life of the new and reconditioned parts and thereto related to it extra costs, is not taken into account.

Chromium plating is one of the most widespread and important galvanic processes. It is the most multy-operated covering in terms of their functional use. Chromium plate harden the tools' face endurance and is used to worn-part reclamation. The technical state of the fuel injection equipment has an effect on oil engines performance. The fuel injection equipment service life is mainly limited by the endurance of the pump elements $[2,8,6]$. The main causes of an accidental release and breaking of the maintenance work are fuel contamination with solid particles, water and gas components.Pump elements are covered with durable and inoxidizing coatings in order to reduce their wear and corrosion. For pump elements maintainability, the most advanced should be such technological methods of recondition, which would minimize the subsequent machining and would allow the process automation. Taken all in all, the recondition method should be carried out at low temperatures in order to avoid warp of parts [1,4,7]. These methods include primarily electrolytic recovery methods.

\section{Results and discussion}


Suspension with plungers covered with chrome, removed from the bath and washed first in the bath with distilled water (for capturing the electrolyte), and then for 1-2 minutes in cold running water. After flushing, plungers are removed from the suspension, free from protective screens and purified from isolation, after which was washed in running water and laid into a special container for plungers.

In the process of chrome, the hydrogen absorption of the chromium layer takes place, which increases its fragility. To remove hydrogen, plungers are kept in the drying cabinet at a temperature of $160-180^{\circ}$ for $2-3$ hours, after which they are cooled in air and exposed to the inspection to determine the quality of the coating.

Chrome coating should be in color transitional from shiny to dairy. On the chrome surface of the plunger should not be peeling, growths, "subagr" or inhomogeneity of color. Partial coating chrome non-working surface is allowed.

The effective use of farming equipment largely depends on the right fit of devices in terms of service parameters conforming to specific operating conditions. The norm of dependability ratio is understood as their limiting value, established by the normative technical documentation. The group norm of dependability ratio is used for product qualification of a given type (brand, model). The use of reconditioned worn elements is an important reserve for reducing the cost of machines repairing in conditions of a short supply of duplicate parts and other raw material resources. However, it is implementedineffectively. The volume of their restoration in recent years has been halved and amounts to $9 \ldots 10 \%$ of the used new duplicate parts. To reduce wear and corrosion of pump elements parts, durable and corrosion-resistant coatings are applied to their surfaces $[3,5,12]$. The elements are preliminarily cleaned, detected, machined to remove wear scars. After that, they are degreased in a tank with caustic-soda solution, sodium carbonate and sodium silicate at $\mathrm{t}=70 \mathrm{C}$. Figure 1 shows a scheme of a fuel pump.

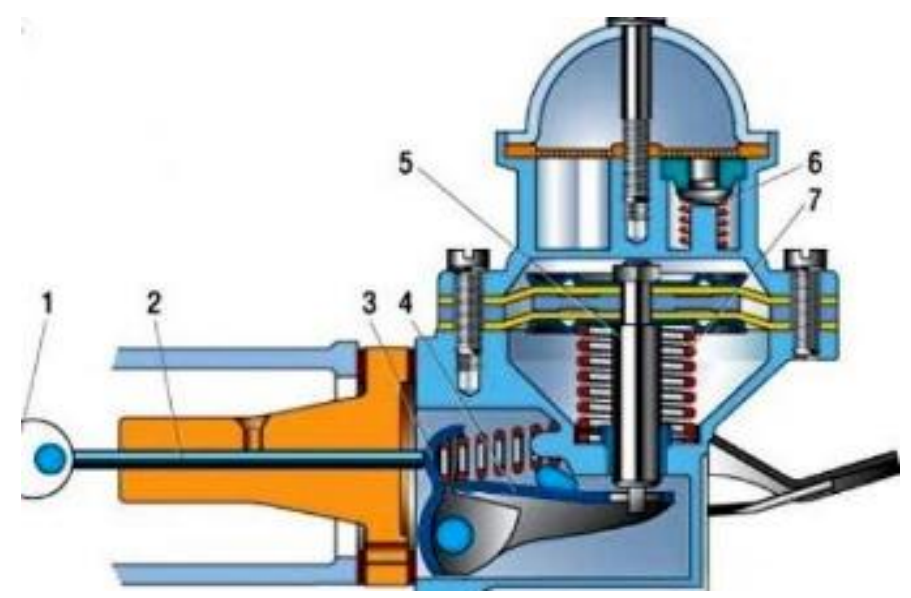

Fig. 1.1 - drive cam; 2 - pusher; 3 - pry bar; 4 - equalizer bar; 5 - stem; 6 - inlet valve; 7 - spring.

In reconditioning the fuel pump plungers with chrome plating with an inner valve diameter of $10 \mathrm{~mm}$. depending on the potential function, the mode and the speed of electrochemical conversions change $[9,10,11,15]$. The latter is illustrated by polarization curves obtained by the potentiostatic method in a chromic acid solution $\left(250 \mathrm{~g} / 1 \mathrm{CrO}_{3}\right)$ with and without the addition of $2.5 \mathrm{~g} / 1 \mathrm{H}_{2} \mathrm{SO}_{4}$ at $18^{\circ} \mathrm{C}$. An increase in the amount of an organic additive drive the chromium plating output up, and the maximum current output of chromium is achievedat its concentration of $2.7 \mathrm{~g} / 1$ (Figure 2). 


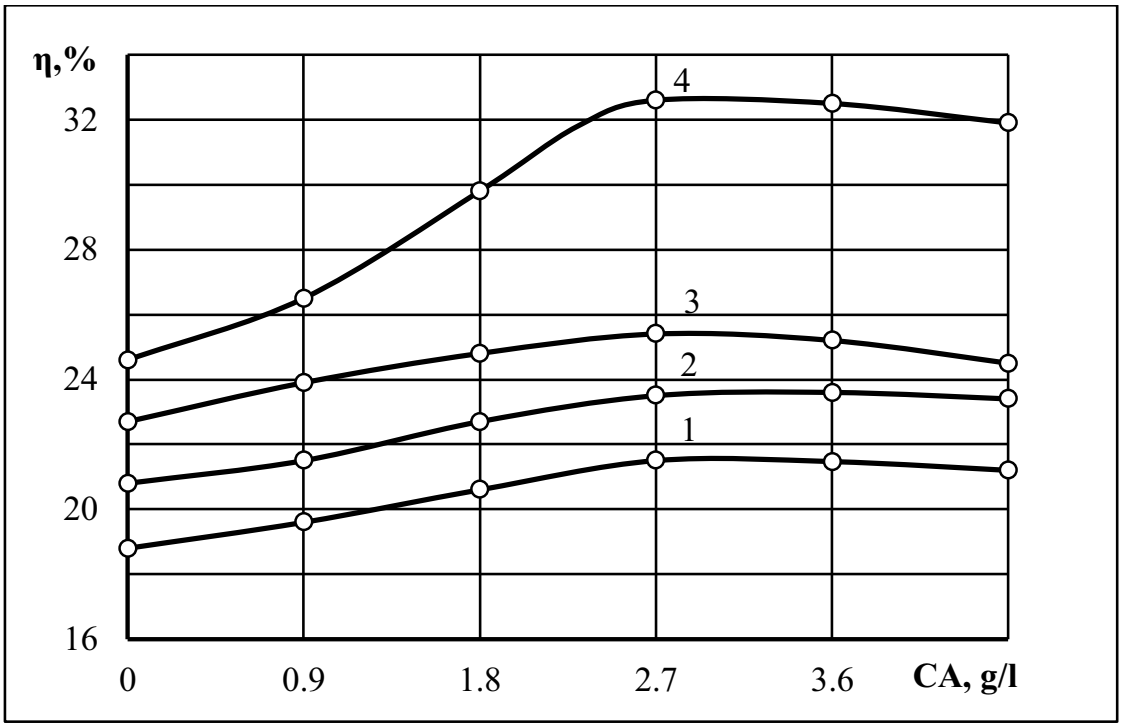

Fig. 2. Effect of the organic additives concentration on the current output of chromium.

$1-\mathrm{D}_{\mathrm{c}}=100 \mathrm{asd} ; \mathrm{t}_{\mathrm{el}}=59^{\circ} \mathrm{C}$;

2- $\mathrm{D}_{\mathrm{c}}=149 \mathrm{asd} ; \mathrm{t}_{\mathrm{el}}=68^{0} \mathrm{C}$;

$3-\mathrm{D}_{\mathrm{c}}=99 \mathrm{asd}^{2} ; \mathrm{t}_{\mathrm{el}}=49^{0} \mathrm{C}$;

4- $\mathrm{D}_{\mathrm{c}}=149 \mathrm{asd} ; \mathrm{t}_{\mathrm{el}}=49^{\circ} \mathrm{C}$;

At the same time, the greatest effect of the additive occures with an increase in the cathodic current density and a fall in the electrolysis temperature. (curve 4). An increase of the electrolyte temperature with this additive reduces the current output of chromium (Figure $3)$.

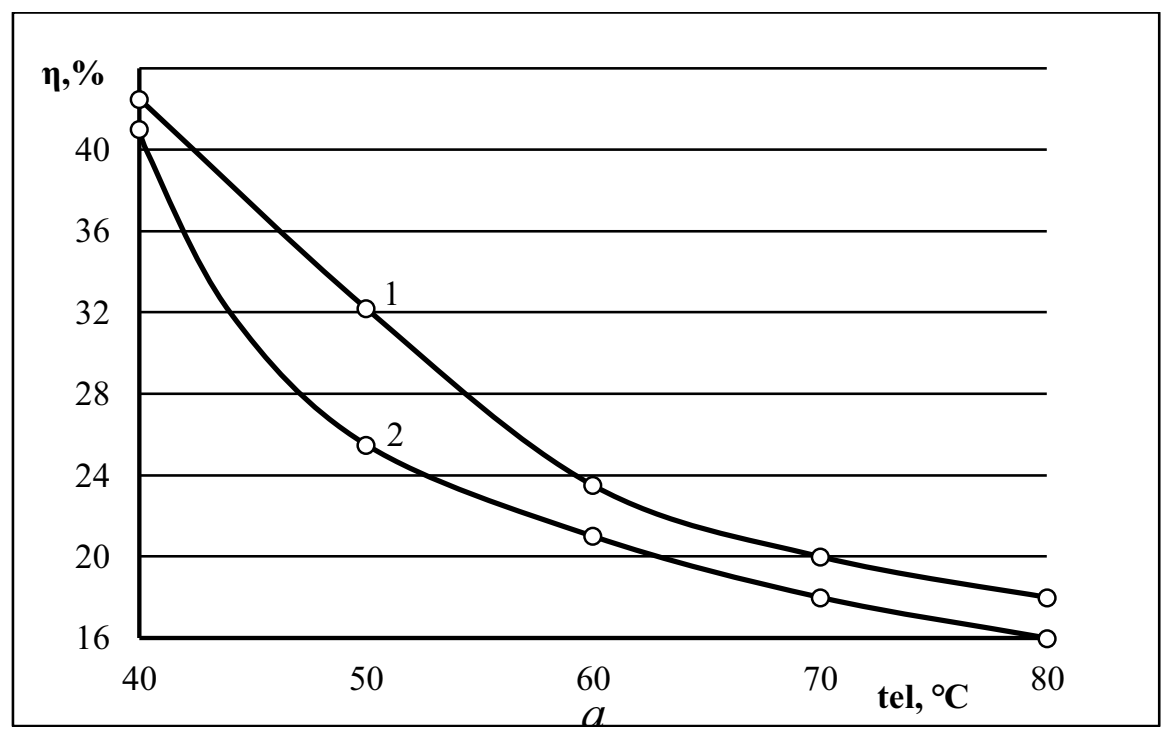

Fig. 3. Effect of the electrolyte temperature on the current output of chromium

These rules are of the same character as in the coating deposition from an electrolyte without additive. Despite the high current output of chromium at $\mathrm{t}=400^{\circ} \mathrm{C}$, it is difficult 
to keep such a temperature condition, because it is necessary to periodically cool the tank down in overlaying a coating to a thickness of more than $50 \mu \mathrm{m}$. At a tentative assessment of the coatings quality of at $\mathrm{t}=400 \mathrm{C}$ and a current density of $100 \ldots 149$ asd a gray set has been obtained. Moreover, the adhesion of the coating with the base reduces. The cathode current density $\left(D_{c}\right)$ has the opposite effect on the change of the chromium output and the plating rate. With its increase, the output of chromium and the plating rate increases (Figure $3 \mathrm{a}, \mathrm{b})$. A similar rule is observed for chromium plating in a electrolyte without additive (curve 2).In an electrolyte with an additive, an intense increase in the current output of chromium occurs up to $D_{c}=149$ asd (Figure 3, a curve 1). Its further increase to 200asd leads to a slowdown output. The use of the additive increases the chromium output by 1.3 1.4 times at $\mathrm{D}_{\mathrm{c}}=49 \mathrm{asd}$, by 1.69 times at $\mathrm{D}_{\mathrm{c}}=149$ asd and $\mathrm{t}_{\mathrm{el}}=490 \mathrm{C}$.

The remanufacturing technique includes cleaning, flaw detection and classifying them into three groups: with wear $0 \ldots 2 \mu \mathrm{m}, 3 \ldots 6 \mu \mathrm{m}, 7$ and more $\mu \mathrm{m}$. After the chromium plating, the components are washed in a trapping tank, then in flowing water. The hangers are opened, the plungers are washed in hot water and dried, heated up in a thermal oven and kept for $60 \ldots 70$ minutes. Then the quality of the coating is monitored, sorted into groups with a diameter difference of $0.005 \mathrm{~mm}$ and grinded. In developing advanced technologies, it is necessary to reduce the labor intensity at the cost of the gross rate of productivity, as well as to boost the rate use of the applied metal coatings. In order to provide consumers with duplicate parts, it is necessary more widely bring in a new process and facilities that allow using worn out component parts.

\section{Conclusion}

Improving the efficiency of repair production in agriculture is closely related to the creation and implementation of such methods for the repair and restoration of parts that allow you to improve the physicomechanical and operational properties of weathered compounds. The resource of the fuel pumps of tractor and combine diesel engines is limited by the performance of plunger pairs, subject to mainly abrasive wear. The presence of an agenic layer on the surface of the parts creates the prerequisites for the preparation of a complex coating based on chromium nitrides.

When recovering plunger pairs of chromium, their wear resistance increases significantly. At the same time, the substantial disadvantage of this method is the uneven coating thickness on plungers, the formation of so-called fungi, which reaches thickness 5 $6 \mathrm{MK}$. In this regard, the subsequent mechanical treatment of chrome plungers is very complicated, which significantly increases the costs of restoring plunger pairs of chromium and reduces the economic efficiency of their recovery.

The porous chromium plating absorbs the lubricant, thereby enabling lubrication without continuous external flow. In the series of voltages, chromium is among the electronegative metals, but thanks to its strong ability to passivation, it takes on the properties of noble metals and shows high resistance to corrosion in atmospheric and chemical conditions. This is due to a passivating oxide slick on its surface, which is easily restored at the slightest damage. Its protective effect is retained at elevated temperatures, which makes it possible to use chrome plating to avoid oxidation of parts operated at temperatures up to $700-$ $800^{\circ} \mathrm{C}$. The tribological tests of chromium-plated specimens have indicated that the wear of specimens covered with a composite chromium-based coating is less than of the specimens coated with a plain electrolyte chromium coating. The seizure load of specimens covered with a composite coating is 1.7 times higher than that ones, applied with electroplated chromium. 


\section{References}

1. V.P. Bagmutov, A.N.Savkin, S.N. Parshev, VSTU. Volgograd, 56 (2011)

2. I.V. Belikov, E.V. Lemeshko, Modern Science, 4-1, 408-413 (2021)

3. T.V. Bodyakina. P.A. Boloev, T.P. Gergenova, and others, Materials of the VIII National Scientific -practical conference with international participation "Readings of I. P. Terskikh", dedicated to the 85th anniversary of the Irkutsk State Agrarian University, Irkutsk, 26-27, 36-41 (2019)

4. O.Y. Erenkov, E.V. Yavorskaya, D.O. Yavorskiy, Repairs. Recovery. Modernization., 4, 3-6 (2020) doi: 10.31044/1684-2561-2020-0-4-3-6

5. L.V. Fedorova, S.K. Fedorov, A.V. Slavin, et al. Metal Science and Heat Treatment of Metals, 2 (776), 58-64 (2020)

6. A.Y. Kozlyuk, V.N. Belyaev, A.S. Andreev, Siberian Scientific Bulletin,1 (25), 73-76 (2019)

7. A.E. Krupin, International Technical and Economic Journal, 2, 112-117 (2017)

8. A.L. Leontiev, L.B. Leontiev, A.G. Toklikishvili, A.I. Shkuratov, Materials of the international research and training conference "Modern materials and technologies", Kursk: South-West. State University, 216-219 (2011)

9. S.V. Malysh, I.M. Kovensky, L.Z. Chaugarova, Proceedings of higher educational institutions, Oil and Gas, 6, 144-149 (2020) doi: 10.31660/0445-0108-2020-6-144-149

10. A.D. Mazitov, Materials of international scientific practical conference of young scientists, Molodezhny settlement, 258-265 (2020)

11. N.P. Ovchinnikov. V.V. Portnyagina, V.I. Dambuev, Journal of the Mining Institute, 241, 53-57 (2020) doi: 10.31897/PMI.2020.1.53

12. S.A. Shishurin, Agrarian academic periodical, 8, 95-99 (2019)

13. S.N. Shukhanov, Baikal Vestnik DAAD, 1, 88-92 (2019)

14. S.N. Shukhanov, A.Y. Kuzkin, V.V. Skutelnik, O.L., Bulletin of the Orenburg State Agrarian University, 1, 107-109 (2018)

15. S.N. Shukhanov, Proceedings of the Orenburg state agrarian University, 1 (75), 74$75(2019)$ 